\title{
$\sigma$ Orionis IRS1 A and B: A Binary Containing a Proplyd
}

\author{
Klaus W. Hodapp ${ }^{1}$, Christof Iserlohe ${ }^{2}$, Bringfried Stecklum ${ }^{3}$, Alfred Krabbe ${ }^{2}$
}

\begin{abstract}
We report optical and infrared imaging spectroscopy observations of the young binary object $\sigma$ Orionis IRS1 A/B. The brighter component ( $\sigma$ Ori IRS1 A) of this binary system has M1 spectral type and a mass in the range of $\approx 0.3-$ $0.8 M_{\odot}$. The fainter component ( $\sigma$ Ori IRS1 B) has a unique morphology and spectrum. The unresolved stellar object is surrounded by an extended envelope that is slightly offset from the position of this star. The envelope's spectrum shows strong emission lines of $\mathrm{H}$ and $\mathrm{HeI}$ but no shock-excited emission from $\mathrm{H}_{2}$ or [FeII]. The embedded stellar object $\sigma$ Ori IRS1 B has an absorption spectrum characteristic of a late $\mathrm{M}$ photosphere, but with an additional approximately equal amount of dust continuum flux veiling the absorption lines. $\sigma$ Ori IRS1 B is probably a young brown dwarf embedded in a proplyd that is being photoevaporated by the UV flux of the nearby multiple $\mathrm{O}$ and B star system $\sigma$ Ori.
\end{abstract}

Subject headings: stars: formation — stars: pre-main sequence — stars: lowmass, brown dwarfs

\section{INTRODUCTION}

The binarity of the late O-star $\sigma$ Ori was discovered by Struve (1837) using visual micrometer measurements. With the further discovery by Bolton (1974) that component $\sigma$ Ori A is itself a spectroscopic binary, the determination of the orbit of components A and B by Heintz (1997) and the identification of the massive C, D, and E components (Caballero 2007a) and (Sherry et al. 2008), the $\sigma$ Ori system is now viewed as somewhat analogous to

\footnotetext{
1 Institute for Astronomy, University of Hawaii, 640 N. Aohoku Place, Hilo, HI 96720, USA email: hodapp@ifa.hawaii.edu

2 Deutsches SOFIA Institut, Universität Stuttgart, Pfaffenwaldring 31, D-70569 Stuttgart, Germany

3 Thüringer Landessternwarte Tautenburg, Sternwarte 5, D-07778 Tautenburg, Germany
} 
the Trapezium system of massive stars in the Orion Nebula. The multiple star system $\sigma$ Ori lies in the center of a small cluster of young lower mass stars, as first discussed by Garrison (1967). This cluster has $\approx 300$ members with a total mass of $150-225 \mathrm{M}_{\odot}$, with ages of individual members ranging from several million years (Sherry et al. 2008) down to stars in the outflow phase (Reipurth et al. 1998) and masses ranging from the massive members of the $\sigma$ Ori group down to objects of possibly only planetary mass (Zapatero Osorio et al. 2008) (and references therein). The properties of the $\sigma$ Ori cluster have recently been reviewed by Walter et al. (2008) and we refer to references therein for more details. Sherry et al. (2008) arrived at a main sequence-fitting distance of $420 \mathrm{pc}$, consistent with the distance to main Orion OB1b association. The extinction towards the $\sigma$ Ori cluster is very low and can be neglected for work in the near infrared.

The discovery by van Loon \& Oliveira (2003) of the infrared object $\sigma$ Ori IRS1 about $3^{\prime \prime}$ north of the high-mass binary $\sigma$ Ori A/B, associated with extended infrared emission and coinciding with a VLA radio source (Drake 1990) raised the possibility that this object is a proplyd, similar to the many objects of this type found in the Orion nebula. $\sigma$ Ori IRS1 has been detected in X-rays by Caballero (2007b) and Skinner et al. (2008). The variability and the high temperature component in its X-ray spectrum are consistent with it containing a magnetically active low mass $\mathrm{T}$ Tauri star. Infrared images using adaptive optics by Bouy et al. (2009) showed that $\sigma$ Ori IRS1 is a double source with components IRS1 A and IRS1 B. In this paper we present near-infrared integral-field spectroscopy of the two components of $\sigma$ Ori IRS1.

\section{OBSERVATIONS AND DATA REDUCTION}

We had noticed the binary nature of $\sigma$ Ori IRS1 independently from Bouy et al. (2009) during observations on 2007, December 30 (UT) using the Keck 2 telescope with the NIRC2 near-infrared camera and natural guide star adaptive optics under non-photometric conditions. The image in the $\operatorname{Br} \gamma$ filter is shown as part of Fig. 1. We observed $\sigma$ Ori IRS1 again with the OH-Suppressing Infrared Integral Field Spectrograph (OSIRIS) (Larkin et al. 2006) at Keck 2 on the night of 2008, Aug. 21, UT, under excellent weather conditions using the finest scale of 20 mas pixel ${ }^{-1}$. The quality of these observations allowed a detailed study of the $\sigma$ Ori IRS1 B emission spectrum in the $H$ and $K$ windows (Figs. 2 and 3) and the spectral classification of both components of the binary star system $\sigma$ Ori IRS1 (Figs. 4). For the $K$-band observations, we used spectra of the multiple $\mathrm{O}$ and $\mathrm{B}$ star system $\sigma$ Ori A/B as the atmospheric absorption standard, after interpolation across the strong $\mathrm{Br} \gamma$ absorption line, and multiplied the ratio spectra by the spectrum of a $30750 \mathrm{~K}$ blackbody, 
the $\mathrm{T}_{\text {eff }}$ of an $09.5 \mathrm{~V}$ star (Massey et al. 2005). We did not obtain calibration spectra of $\sigma$ Ori A/B in the $H$-band. Therefore, the $H$-band spectrum of $\sigma$ Ori IRS1 B in Fig. 2 used the M1 star $\sigma$ Ori IRS1 A as the atmospheric absorption standard, and the ratio spectrum was multiplied by the spectrum of the M1V star HD42581 from the spectral library of Cushing, Rayner, \& Vacca (2005), to establish the proper continuum. The spectrum in Fig. 2 was separately flux calibrated in the $H$ and $K$ bands by multiplying the spectrum with the 2MASS spectral response curves, integrating the flux, using the 2MASS flux calibration

of Cohen. Wheaton, \& Megeath (2003), and then scaling the result to match the published $H$ and $K s$ magnitudes of Bouy et al. (2009).

We also obtained optical seeing-limited slit-less spectroscopy using the Wide Field Grism Spectrograph 2 (WFGS2) (Uehara et al. 2004) at the UH 2.2m telescope in an effort to detect the $\mathrm{H} \alpha$ line from $\sigma$ Ori IRS1 B. The images were obtained on the night of 2008, October 11, UT, under near-photometric conditions. This wide-field collimator-camera system was used without a slit, with a narrow-band $\mathrm{H} \alpha$ filter (10 nm FWHM) as a spectral selector, and with the high-resolution grating, resulting in a dispersion of $0.085 \mathrm{~nm} \mathrm{pixel}{ }^{-1}$. As a result, all stars are dispersed into short continua, covering the transmission range of the $\mathrm{H} \alpha$ filter. The central area of the resulting image is shown in Fig. 1. The emission-line dominated $\sigma$ Ori IRS1 B is easily seen in the resulting slit-less spectroscopy image as the only object near the $\sigma$ Ori system that appears as an unresolved dot of $\mathrm{H} \alpha$ emission, without detectable continuum or velocity structure.

\section{SPECTRAL CLASSIFICATION OF IRS1 A AND B}

The median age of the $\sigma$ Ori cluster is 2-3 Myr (Sherry et al. 2008), so that all low mass objects are still contracting, and are therefore larger, more luminous, and have lower surface gravity than main sequence stars. When attempting to classify their spectra, it is usually found that their features fall between those of luminosity class V and III, but closer to class V, as was shown in the early work by Hodapp \& Deane (1993) and Greene \& Lada (1996). In mid to late $\mathrm{M}$ spectral types, the ratio of the strength of the $\mathrm{CO}$ bandheads to that of the atomic lines, as well as the ratio of the two components of the Na doublet (see Fig. 4), are luminosity dependent and the latter show that both $\sigma$ Ori IRS1 components are indeed much closer to luminosity class $\mathrm{V}$ than to class III.

The spectral classification of the two components of $\sigma$ Ori IRS1 relies on the strongest molecular feature in the atmospheric $K$ window, the CO bandheads, and on the atomic line systems of $\mathrm{Na}, \mathrm{Ca}, \mathrm{Mg}$, and the complex of $\mathrm{Fe}$ and $\mathrm{Ti}$ lines at $2.23 \mu \mathrm{m}$. The spectral classification was done by discussion of features in direct comparison (Fig. 4) with the IRTF/SPEX 
spectral library of Cushing, Rayner, \& Vacca (2005).

\section{1. $\sigma$ Ori IRS1 A}

In the spectrum of IRS1 A in Fig. 4 the absorption line systems of $\mathrm{Na}$ and $\mathrm{Ca}$ are of almost equal strength. The absorption line of $\mathrm{Mg}$ at $2.282 \mu \mathrm{m}$ is clearly detected. This is most closely matched by the comparison spectrum of M1V type that also reproduces the shape of the Fe and Ti complex at $2.23 \mu \mathrm{m}$ best. For later spectral types (M3V), the Mg line is too weak and the shortest wavelength component of the Ca feature becomes too strong. For even earlier spectral types, $\mathrm{Mg}$ becomes too strong, and the line ratios of the $\mathrm{Ca}$ and the Fe and Ti multiplets do not match $\sigma$ Ori IRS1 A. The CO bandheads in IRS1 A are stronger compared to $\mathrm{Na}$ and $\mathrm{Ca}$ than in M1V spectral types, as it would be expected for an object of higher than main sequence luminosity. We conclude that a spectral type of M1V is the closest match to the spectrum of IRS1 A with an uncertainty of one decimal sub-class, the caveat being that IRS1 A is more luminous than a main sequence object and that it is young. Following Luhman et al. (2003), this gives its effective temperature as $3705 \pm 150 \mathrm{~K}$.

From the $\mathrm{K}_{s}$ magnitude of 10.48 (Bouy et al. 2009) and with colors and bolometric corrections from Johnson (1965), we arrive at a rough estimate for the luminosity of $\sigma$ Ori IRS1 A of $0.86 L_{\odot}$. Comparison of these $\mathrm{T}_{\text {eff }}$ and $\mathrm{L}$ data with the 1998 revisions of the D'Antona \& Mazzitelli (1997) evolutionary tracks in Fig. 5 places $\sigma$ Ori IRS1 A on the track of a $0.3 \mathrm{M}_{\odot}$ star with a nominal age of 0.3 Myrs. Compared to the 1 Myr isochrone of the Baraffe et al. (1998) and Chabrier et al. (2000) models $\sigma$ Ori IRS1 A appears to be a star of $0.8 \mathrm{M}_{\odot}$ and an age of $\approx 1 \mathrm{Myr}$. The errors indicated in Fig. 5 represent the uncertainty of \pm 1 subclass in spectral type determination, and the estimated total error from the uncertainty in bolometric corrections values (Malkov et al. 1997), color transformations, and photometric errors. As discussed both by D’Antona \& Mazzitelli (1997) and Baraffe et al. (2002), the evolutionary models themselves are still quite uncertain at these young ages, adding another caveat to the interpretation of the results.

\section{2. $\sigma$ Ori IRS1 B}

The best fit to the line ratios of $\sigma$ Ori IRS1 B is a M7 or M8 star on the basis of the detection of $\mathrm{Na}$ lines and non-detection of $\mathrm{Ca}$ lines, and the ratio of $\mathrm{Na}$ to $\mathrm{CO}$ bandhead strength. However, all these late $\mathrm{M}$ class $\mathrm{V}$ stars have much stronger lines (both the $\mathrm{Na}$ and the CO bandhead lines) than $\sigma$ Ori IRS1 B, so in order to match the spectrum, we have to 
postulate an additional, diluting continuum component to the spectrum. As we will discuss in detail in Section 4. the strong spatially extended $\operatorname{Br} \gamma$ and HeI emission suggests that $\sigma$ Ori IRS1 B is a proplyd in the process of being photo-evaporated by UV radiation from the OB stars in the $\sigma$ Ori A/B system. It is therefore reasonable to assume that some dust in the proplyd gets heated by UV absorption to temperatures near the sublimation point, and that its emission dilutes the photospheric emission from the embedded star. However Fig. 3 clearly shows that the line emission is more extended than the continuum, which appears unresolved. It is also possible that part of the hot dust emission originates much closer to the star and is powered by an accretion disk. Our data are not sufficient to uniquely identify a combination of dust opacity and temperature to best match the spectrum, but solutions with dust temperatures in the range of $1000 \mathrm{~K}$ and contributions of about $50 \%$ of dust emission to the total flux give good matches to the line depths. Fig. 4 therefore compares the spectrum of $\sigma$ Ori IRS1 B, expanded by a factor of 2 to account for the dilution of the lines, with archival spectra of mid-M stars (above) and M8-L1 stars and an M7III star below. This comparison places the spectral type of $\sigma$ Ori IRS1 B in the range from M6 to M9. A spectral type as early as M6 is consistent with the data when we consider that for lower gravity atmospheres, the $\mathrm{Na}$ is fainter and the $\mathrm{CO}$ bandheads are stronger than for main sequence stars. On the other extreme, the M9 class is still compatible with the data, considering the substantial errors. A spectral type of M5 can be excluded with confidence, since the Ca feature would be much too strong, and on the other side, a L1 or later type can be excluded since those types do not show Na absorption. We therefore nominally classify $\sigma$ Ori IRS1 B as M7.5, i.e., the midpoint of the range of possible spectral types. We assume as the effective temperature of $\sigma$ Ori IRS1 B the average of the effective temperatures given for M7 and M8 in Luhman et al. (2003): $2795 \mathrm{~K}$ with a range of uncertainty between $2400 \mathrm{~K}$ (M9V) and $3000 \mathrm{~K}(\mathrm{M} 6 \mathrm{~V})$. While it is clear from its luminosity and age that $\sigma$ Ori IRS1 B is not a main sequence star, its spectrum does not show, within the limitations of the signalto-noise ratio, any specific features related to lower surface gravity. Nevertheless, it should be classified as luminosity class IV.

With a measured $K_{s}=12.65$ from Bouy et al. (2009), we obtain an $\mathrm{M}_{b o l}$ of 7.10 using colors and bolometric corrections from Johnson (1965) and a luminosity of $\mathrm{L}=0.109 \mathrm{~L}_{\odot}$. The errors indicated in Fig. 5 represent the uncertainty in the determination of the spectral type, and the maximum effect of a factor of 2 in luminosity from either including or excluding the dust emission in the determination of the luminosity. It places $\sigma$ Ori IRS1 B on the evolutionary track of a $0.10 M_{\odot}$ object in the 1998 revisions of the D'Antona \& Mazzitelli (1997) models (Fig. 5) near the $0.3 \mathrm{Myr}$ age isochrone. We also plot the 1 Myr isochrone of the models by Baraffe et al. (1998) and Chabrier et al. (2000) and note that $\sigma$ Ori IRS1 B is well above this isochrone. The differences between both models illustrate the problems 
with theoretical HR diagrams at young ages that the authors of those models are well aware of (Baraffe et al. 2002). As an alternative to comparing with evolutionary models, we can compare $\sigma$ Ori IRS1 B directly to the components of young the eclipsing binary 2MASSJ05352184-0546085 in the Orion Nebula whose masses have been directly measured by Stassun, Mathieu, \& Valenti (2006). This star's primary component of M6.5 \pm 0.5 spectral type has a measured mass of $0.0541 M_{\odot}$ while the slightly hotter secondary component has a mass of $0.0340 M_{\odot}$. Since $\sigma$ Ori IRS1 B is of later spectral type than either of the components of this eclipsing binary and is probably of similar age, it seems certain that $\sigma$ Ori IRS1 B has a sub-stellar mass below $0.05 M_{\odot}$. Finally, in a strong UV radiation environment, the photospheric temperature may be elevated above what an isolated object of otherwise identical properties might have, which would lead to an overestimate for the mass.

\section{THE EMISSION LINE SPECTRUM OF $\sigma$ ORI IRS1 B}

The emission line spectrum in the $H$ and $K$ bands of $\sigma$ Ori IRS1 B is shown in Fig. 2. The spectrum is dominated by strong emission from the Brackett series of $\mathrm{HI}$ and by emission lines of HeI. There is, however, no detectable emission from shock-excited $\mathrm{H}_{2}$ in the $\mathrm{S}(1)$ lines, nor is there forbidden line emission in the $1.644 \mu \mathrm{m}$ emission line of [FeII], which is often associated with outflow activity in young stars. Emission lines are one of the defining characteristics (Jov 1945) of classical T Tauri stars and in those stars originate from the accretion disk. In the case of $\sigma$ Ori IRS1 B, however, our spatially resolved line images show that the $\mathrm{Br} \gamma$ and HeI emission lines do not originate in an accretion disk very close to the stellar surface. Rather, the line emission is spatially extended by 95 mas FWHM in the case of the $\mathrm{Br} \gamma$ line, and similar for HeI, while the PSF is 55 mas FWHM. The brightest emission is offset towards the illuminating $\mathrm{OB}$ stars as illustrated in Fig. 3. On the other hand, the emission lines do not show a pronounced bow-shock morphology, indicating that we are not seeing IRS1 B being illuminated side-on. This finding agrees with the morphology of the 10 $\mu \mathrm{m}$ emission found by van Loon \& Oliveira (2003) who did not see the bow shock structure often found at $10 \mu \mathrm{m}$ in side-on Orion proplyds by Smith (2005).

While the proplyd IRS1 B was easily detected in $\mathrm{H} \alpha$ emission, its surface brightness falls well short of the prediction by van Loon \& Oliveira (2003) of being equal to the $\sigma$ Ori A/B continuum in a $1 \mathrm{~nm}$ wavelength interval. Calibrating the continuum flux of $\sigma$ Ori A/B against the spectrophotometry by Stone (1996) of the HST O9Vp calibration star HD93521, we get an $\mathrm{H} \alpha$ flux for the unresolved $\mathrm{H} \alpha$ emission knot from $\sigma$ Ori IRS1 B of $4.710^{-15} \mathrm{Wm}^{-2}$. The dust that van Loon \& Oliveira (2003) detected in thermal emission probably absorbs 
much the $\mathrm{H} \alpha$ emission that would be expected based on extrapolation from the radio spectrum.

\section{DISCUSSION}

The formation of low-mass objects by photo-evaporation of prestellar cores near massive stars has been discussed by Whitworth \& Zinnecker (2004), and our data indicate that $\sigma$ Ori IRS1 B appears to show this process in action. It is interesting to note that the two components of the $\sigma$ Ori IRS1 system show very different spectra. The more luminous component IRS1 A shows a pure photospheric absorption line spectrum without evidence for dust veiling or emission lines. Assuming that the two components are indeed physically close to each other, and that IRS1 A is therefore exposed to the same intense UV radiation field as IRS1 B, it can be concluded that IRS1 A must be without a disk or envelope. While distant encounter between stars in the dense cores of clusters are efficient at stripping the

outer parts of disks (Pfalzner, Umbreit, \& Henning 2005), the complete lack of any sign of circumstellar matter around the more massive component A of the IRS1 system can only be explained by dynamical effects within that system. It can be speculated that IRS1 A itself might be a very close binary whose formation may have destroyed the disk, in a scenario similar to those discussed by Reipurth (2000).

$\sigma$ Ori IRS1 B is not the only object undergoing photoevaporation in the UV radiation field of $\sigma$ Ori A and B. In a recent paper, Rigliaco et al. (2009) showed that the T Tauri star SO587 in the $\sigma$ Ori cluster shows emission lines that can best be interpreted in a photoevaporation scenario.

\section{CONCLUSIONS}

We have obtained optical and near-infrared spectroscopy of the binary object $\sigma$ Ori IRS1 A and B. Component $\sigma$ Ori IRS1 A shows a pure photospheric absorption line spectrum of M1 type and is a low mass star. Component B of the $\sigma$ Ori IRS1 binary shows a compound dust and photospheric spectrum. After accounting for the dust continuum veiling, $\sigma$ Ori IRS1 B itself can be classified as a M7 or M8 spectral type and probably is of sub-stellar mass. Further, IRS1 B shows a strong emission line spectrum with the Brackett series of $\mathrm{H}$ and HeI lines in the near infrared, and $\mathrm{H} \alpha$ in the optical. The line images in the $\mathrm{Br} \gamma$ and HeI $(2.06 \mu \mathrm{m})$ lines show that the source of the line emission is an extended envelope around the star. This envelope of $\sigma$ Ori IRS1 B is clearly being photo-evaporated by the UV radiation 
field of $\sigma$ Ori A.

We thank Bo Reipurth for helpful discussions.

Some of the data presented herein were obtained at the W.M. Keck Observatory, which is operated as a scientific partnership among the California Institute of Technology, the University of California and NASA. The Observatory was made possible by the generous financial support of the W.M. Keck Foundation. 


\section{REFERENCES}

Baraffe, I., Chabrier, G., Allard, F., \& Hauschildt, P. H. 1998, A\&A, 337, 403

Baraffe, I., Chabrier, G., Allard, F., \& Hauschildt, P. H. 2002, A\&A, 382, 563

Bolton, C. T. 1974, ApJ, 192, L7

Bouy, H., Huélamo, N., Martín, E. L., Marchis, F., Barrado y Navascués, D., Kolb, J., Marchetti, E., Petr-Gotzens, M. G., Sterzik, M., Ivanov, V. D., Köhler, R., \& Nürnberger 2009, A\&A, 493, 931

Chabrier, G., Baraffe, I., Allard, F., \& Hauschildt, P. 2000, ApJ, 542, 464

Caballero, J. A. 2007, AN 328, 1

Caballero, J. A. 2007, A\&A, 466, 917

Cohen, M., Wheaton, Wm. A., \& Megeath, S. T. 2003, AJ, 126, 1090

Cushing, M. C., Rayner, J. T., \& Vacca, W. D. 2005, ApJ, 623, 1115

D’Antona, F. \& Mazzitelli, I. 1994, ApJS, 90, 467

D’Antona, F. \& Mazzitelli, I. 1997, Mem. Soc. Astr. Ital., 68, 807

Drake, S. A. 1990, AJ, 100, 572

Garrison, R. F., 1967, PASP, 79, 433

Greene, T. P. \& Lada, C. J. 1996, AJ, 112,2184

Heintz, W. D. 1997, ApJS, 111, 335

Hodapp, K.-W., \& Deane, J. 1993, ApJS, 88, 119

Johnson, H. L. 1965, ApJ, 141, 170

Joy, A. H. 1945, ApJ, 102, 168

Kleinmann, S. G., \& Hall, D. N. B. 1986, ApJS, 62, 501

Larkin, J. et al. 2006, New Astr. Reviews, 50, 362

Luhman, K. L., Stauffer, J. R., Muench, A. A., Rieke, G. H., Lada, E. A., Bouvier, J., \& Lada, C. J. 2003, ApJ, 593, 1093 
Malkov, O. Yu., Piskunov, A. E., \& Shpil'kina, D. A. 1997, A\&A, 320, 79

Massey, P., Puls, J., Pauldrach, A. W. A., Bresolin, F., Kudritzki, R. P., \& Simon, T. 2005, ApJ, 627, 477

Pfalzner, S., Umbreit, S., \& Henning, Th. 2005, ApJ, 629, 526

Reipurth, B., Bally, J., Fesen, R. A., \& Devine, D. 1998, Nature, 396, 343

Reipurth, B. 2000, AJ, 120, 3177

Rigliaco, E., Natta, A., Randich, S., \& Sacco, G. 2009, A\&A, in press (arXiv:0902.0457v1)

Sherry, W. H., Walter, F. M. , Wolk, S. J. \& Adams, N. R. 2008, AJ, 135, 1616

Skinner, S. L., Sokal, K. R., Cohen, D. H., Gagné, M., Owocki, S. P., \& Townsend, R. D. 2008, ApJ, 683, 796

Skrutskie, M. F., Cutri, R. M., Stiening, R., Weinberg, M. D., Schneider, S., Carpenter, J. M., Beichman, C., Capps, R., Chester, T., Elias, J., Huchra, J., Liebert, J., Lonsdale, C., Monet, D. G., Price, S., Seitzer, P., Jarrett, T., Kirkpatrick, J. D., Gizis, J., Howard, E., Evans, T., Fowler, J., Fullmer, L., Hurt, R., Light, R., Kopan, E. L., Marsh, K. A., McCallon, H. L., Tam, R., Van Dyk, S., \& Wheelock, S. 2006, AJ, 131, 1163

Smith, N., Bally, J., Shuping, R. Y., Morris, M., \& Kassis, M. 2005, AJ, 130, 1763

Stassun, K. G., Mathieu, R. D., \& Valenti, J. A. 2006, Nature, 440, 311

Stone, R. P. S. 1996, ApJS, 107, 423

Struve, F. G. W. 1837, Astronomische Nachrichten, 14, 249

Uehara, M., et al. 2004, Proc. SPIE, 5492, 661

van Loon, J. Th., Oliveira, J. M. 2003, A\&A, 405, L33

Walter, F. M., Sherry, W. H., Wolk, S. J., \& Adams, N. R. 2008, in Handbook of Star Forming Regions, Vol. I, Astronomical Society of the Pacific, Bo Reipurth, ed., p. 732.

Whitworth, A. P. \& Zinnecker, H. 2004, A\&A, 427, 299 
Zapatero Osorio, M. R., Béjar, V. J. S., Bihain, G., Martín, E. L., Rebolo, R., Villó-Pérez, I., Díaz-Sánchez, A., Pérez Garrido, A., Caballero, J. A., Henning, T., Mundt, R., Barrado y Navascués, D., \& Bailer-Jones, C. A. L. 2008, A\&A, 477, 895

This preprint was prepared with the AAS LATEX macros v5.2. 

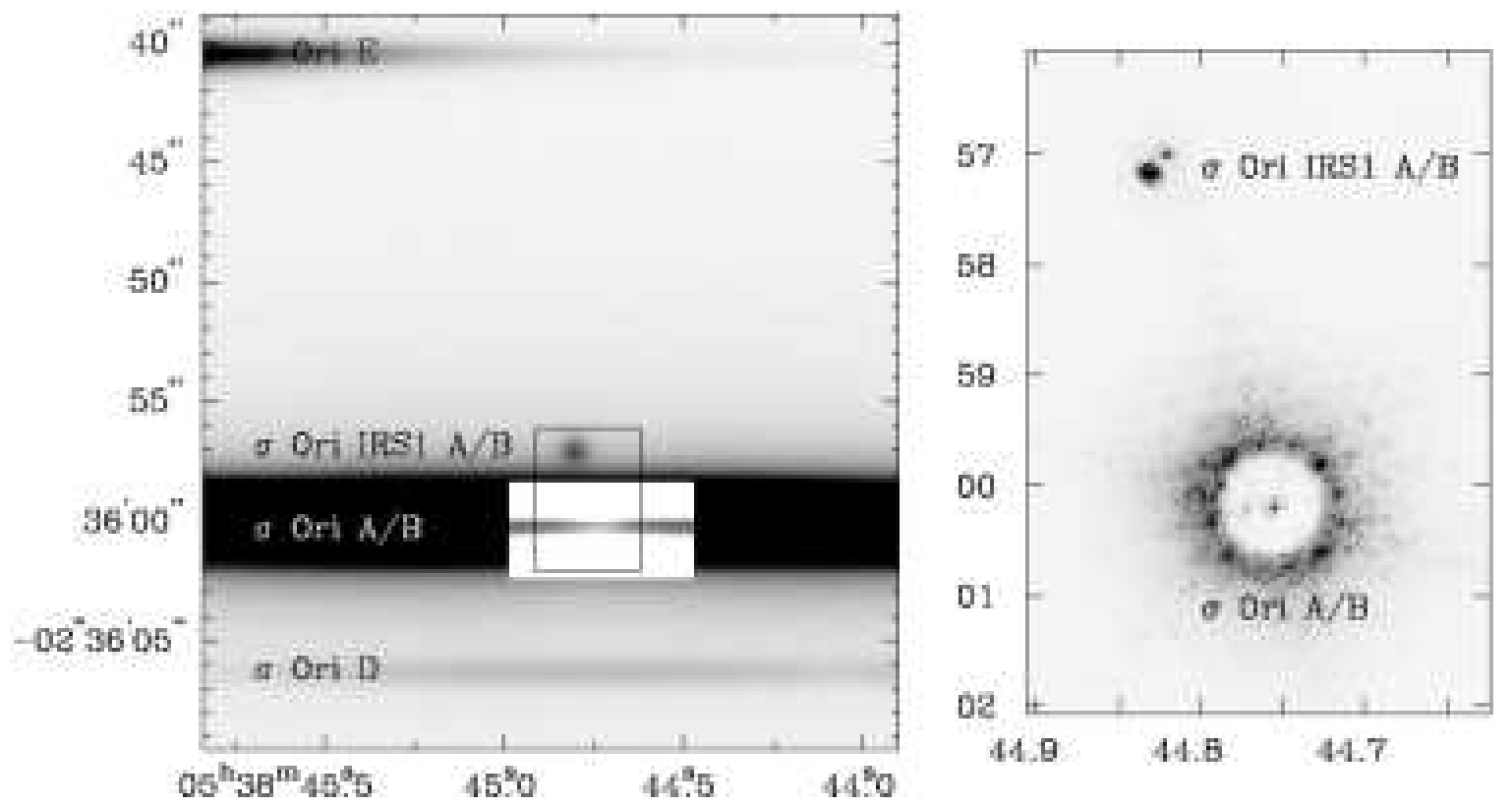

Fig. 1. - Slit-less spectrum of the $\sigma$ Ori system, pre-filtered and centered around H $\alpha$. The brightest continuum is the binary $\sigma$ Ori A/B with its late $\mathrm{O}$ to early $\mathrm{B}$ compound spectrum, with additional contamination from component $\sigma$ Ori $\mathrm{C}$. The spatial coordinates refer to the wavelength of $\mathrm{H} \alpha$. The $\mathrm{H} \alpha$ absorption of the binary $\sigma$ Ori A/B is clearly seen in the higher signal level window. The proplyd $\sigma$ Ori IRS1 B is the object detected only by its $\mathrm{H} \alpha$ emission line to the north of the bright binary. The image to the right is the Keck NIRC-2 adaptive optics image of the $\sigma$ Ori system in the $\operatorname{Br} \gamma+$ continuum filter, covering the area indicated by the box in the $\mathrm{H} \alpha$ image. The main components $\sigma$ Ori A/B are visible behind the partly transparent occulting mask. 


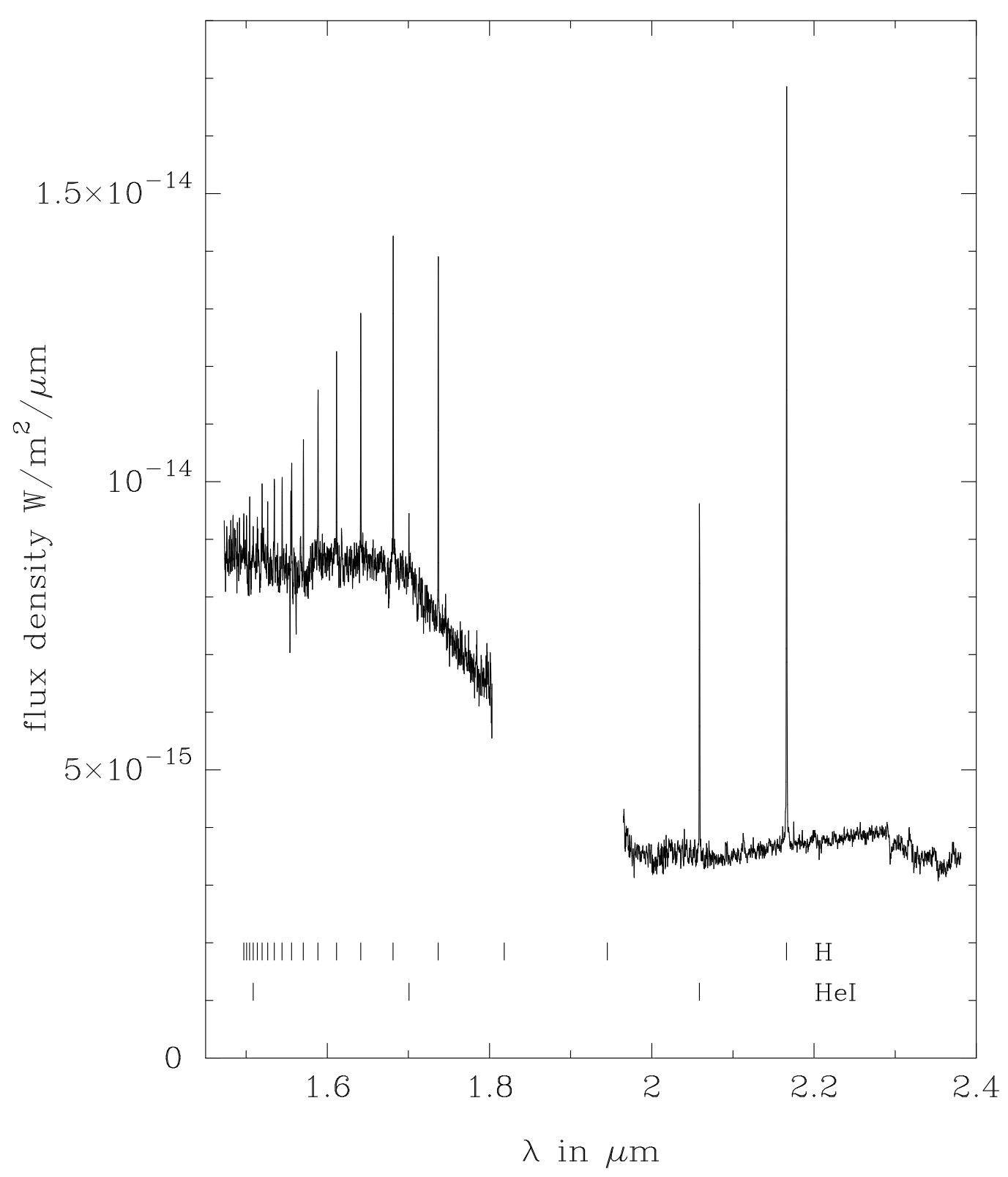

Fig. 2.- Spectrum of $\sigma$ Ori IRS1 B in the $H$ and $K$ bands, showing emission lines of $\mathrm{H}$ and HeI superposed over a continuum spectrum composed of a M7.5 photosphere and dust continuum veiling. 


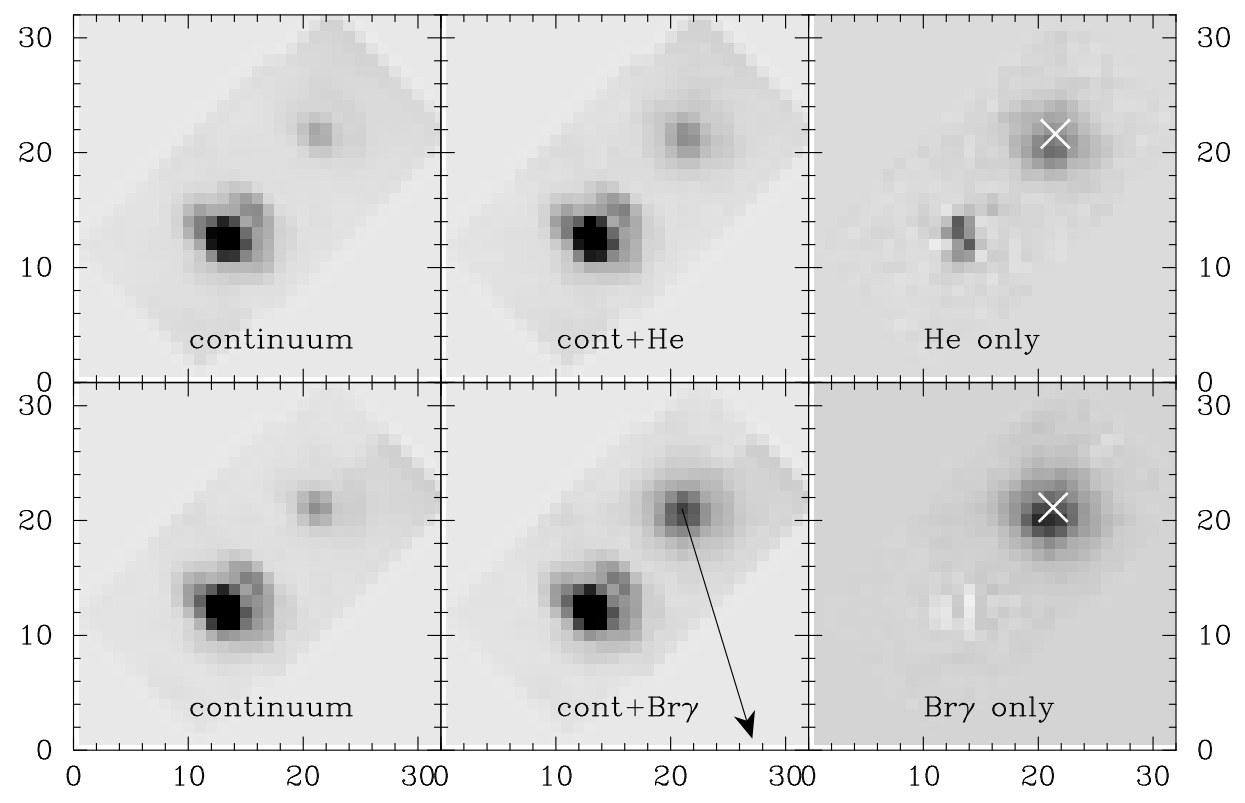

Fig. 3.- Extracted adjacent continuum, line plus underlying continuum, and continuumsubtracted line images of $\sigma$ Ori IRS1 A and B in the emission lines of HeI $(2.059 \mu \mathrm{m})$ and H $\operatorname{Br} \gamma(2.166 \mu \mathrm{m})$. Tickmarks are in units of the OSIRIS 20 mas pixels. The arrow indicates the direction towards the illuminating UV source $\sigma$ Ori A/B. The X symbol in the continuumsubtracted images indicates the position of the star. It should be noted that $\sigma$ Ori IRS1 A is unresolved and that all the structure seen around it is due to the PSF. 


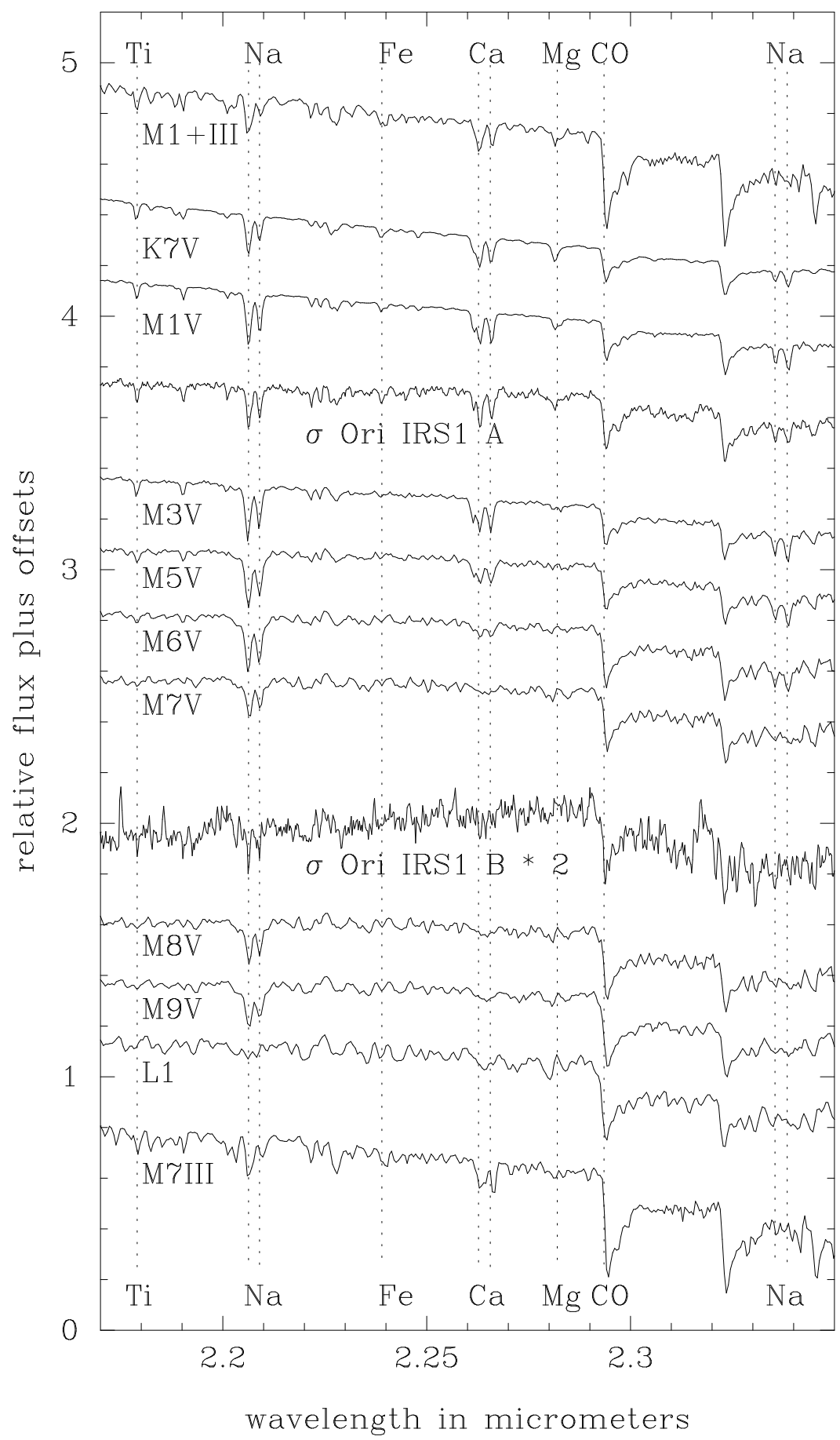

Fig. 4.- Continuum spectra of $\sigma$ Ori IRS1 A and IRS1 B in the $K$ window and comparison spectra from the IRTF spectral library (Cushing, Rayner, \& Vacca 2005). 


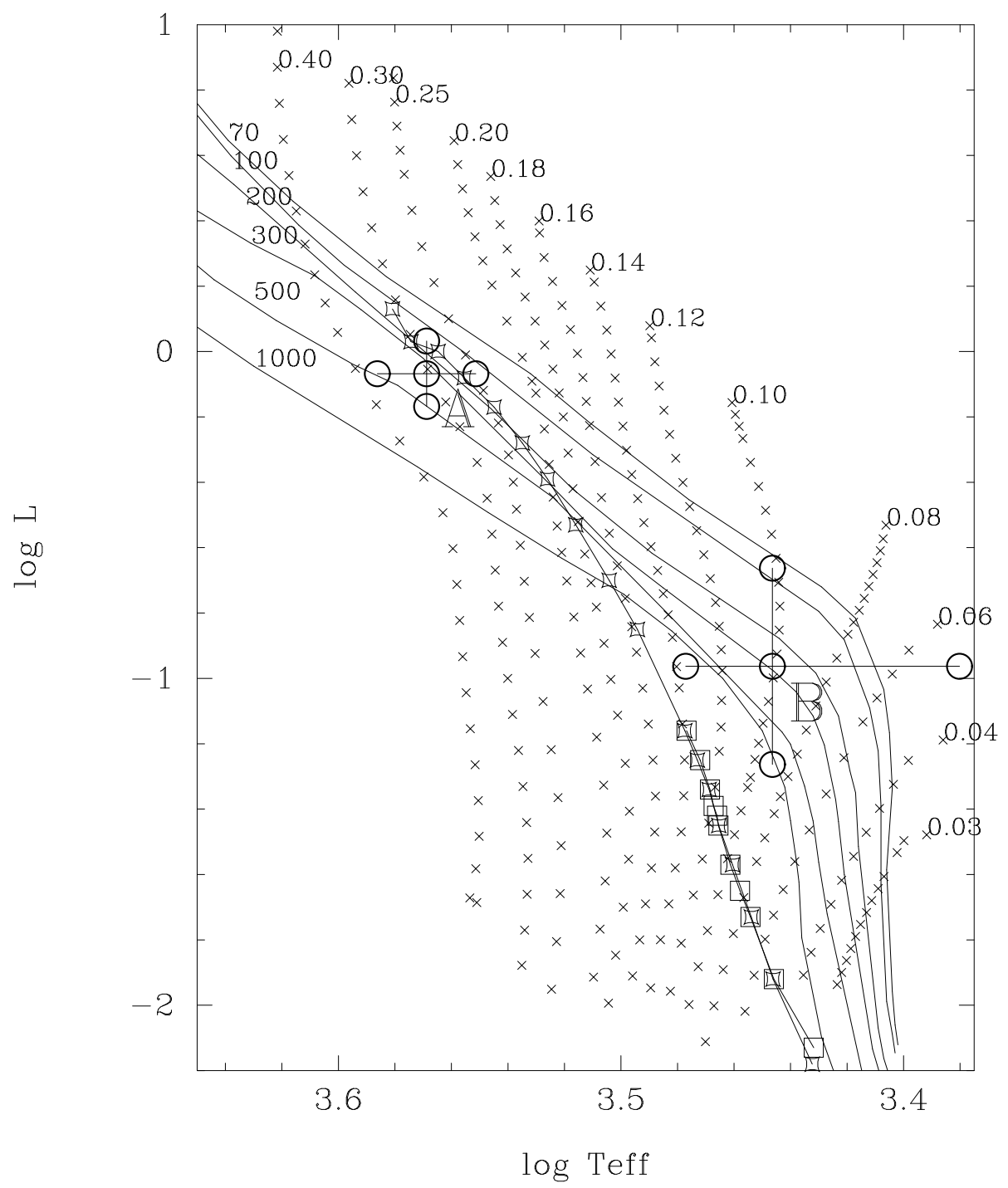

Fig. 5.- Pre-main-sequence evolutionary tracks from D'Antona \& Mazzitelli (1997) (x symbols). Their Isochrones are labeled in units of $1000 \mathrm{yrs}$. The $1 \mathrm{Myr}$ isochrone from Baraffe et al. (1998) and Chabrier et al. (2000) is indicated by open squares. The HR diagram loci of $\sigma$ Ori IRS1 A and B are indicated. The error bars indicate the errors in spectral type determination and photometric errors. 